\section{Fatores associados ao uso regular de cigarros por adolescentes estudantes de escolas públicas de Londrina, Paraná, Brasil}

\author{
Factors associated with regular cigarette smoking \\ by adolescents from public schools in Londrina, \\ Paraná, Brazil
}

\author{
Factores asociados con el consumo regular de \\ tabaco en adolescentes de escuelas públicas \\ en Londrina, Paraná, Brasil
}

Centro de Ciências da
Saúde, Universidade
Estadual de Londrina,
Londrina, Brasil.
2 Autarquia Municipal
de Saúde de Londrina,
Prefeitura Municipal de
Londrina, Londrina, Brasil.
3 Centro de Ciências Exatas,
Universidade Estadual de
Londrina, Londrina, Brasil.
4 Department of Medicine,
University of Alabama,
Birmingham, U.S.A.
5 Centro de Ciências da
Saúde, Universidade
Estadual de Maringá,
Maringá, Brasil.
Correspondência
A. R. H. Menezes
Departamento de Atenção
Primária em Saúde, Centro
de Ciências da Saúde,
Universidade Estadual de
Londrina.
Rua Flor do Paraíso 80,
Londrina, PR
86035-25o, Brasil.
menezessgtbm@yahoo.com.br

Abstract

This cross-sectional epidemiological study aimed to identify factors associated with regular cigarette smoking among adolescents. The sample included 517 public school 9th graders in Londrina, Paraná State, Brazil. A structured questionnaire was used to collect information, including social and demographic characteristics, social relations, and health risk behaviors. Logistic regression was used in the statistical analysis, with significance set at 5\%. $8.9 \%$ of the adolescents reported smoking at least one cigarette a day in the previous 30 days. Age $\geq 15$ years $(O R=2.28 ; 95 \% C I: 1.21-4.32 ; p=0.011)$ and having friends that smoked $(O R=12.62$; 95\%CI: 4.44-35.89; $p<0.0001$ ) were associated with regular smoking in both the univariate and multivariate analyses. Meanwhile, gender, race, social class, living with father and mother, religion, maternal education, having tried alcohol, physical activity, and paid work were not associated with smoking. These findings support the development of community-based tobacco control strategies targeting adolescents.

Smoking; Tobacco; Students; Adolescent Health

\author{
Adriana Henriques Ribeiro Menezes 1,2 \\ José Carlos Dalmas 3 \\ Isabel Cristina Scarinci 4 \\ Sandra Mara Maciel 5 \\ Alexandrina Aparecida Maciel Cardelli 1
}

\section{Resumo}

Estudo epidemiológico transversal com objetivo de identificar fatores associados ao uso regular de cigarros por adolescentes. A amostra foi constituída por 517 alunos do 9o ano do Ensino Fundamental de escolas públicas de Londrina, Paraná, Brasil. O instrumento de coleta de dados incluiu características sociodemográficas, relações sociais e comportamentos de risco. Na análise estatística, foi utilizado o modelo de regressão logística com nível de significância em 5\%. Relataram uso de cigarros, pelo menos, um dia nos últimos trinta dias 8,9\% dos alunos. Os fatores idade $\geq 15$ anos (OR =2,28; IC95\%: 1,21-4,32; $p=0,011)$ e possuir amigos fumantes $(O R=12,62$ IC95\%: 4,44-35,89; $p<0,0001$ ) associaram-se ao uso regular de cigarros, tanto na análise univariada como na multivariada. Por outro lado, sexo, raça, classe social, morar com pai e mãe, religião, escolaridade materna, experimentação de álcool, atividade física e atividade remunerada não se associaram a essa prática. Esses achados podem subsidiar a elaboração de estratégias antitabágicas direcionadas à comunidade, tendo o adolescente como alvo.

Hábito de Fumar; Tabaco; Estudantes; Saúde do Adolescente 


\section{Introdução}

As diversas estratégias de controle desenvolvidas nas últimas décadas resultaram no declínio do número de fumantes. $\mathrm{O}$ acesso, cada vez mais precoce, a produtos derivados do tabaco causa um aumento progressivo do número de jovens fumantes 1 .

O uso do tabaco na adolescência torna urgente a elaboração de propostas e implementação de ações específicas. A Organização Mundial da Saúde (OMS) prevê que, diariamente, cerca de 100 mil crianças e adolescentes tornam-se fumantes em todo o mundo 2 . Sabe-se que $90 \%$ dos adultos fumantes iniciaram o hábito na adolescência, e $75 \%$ dos que começam a fumar na adolescência mantêm hábitos tabagistas na fase adulta 1,2,3. Recente estudo brasileiro apontou que $77,1 \%$ dos fumantes adultos iniciaram o hábito de fumar diariamente antes dos 19 anos de idade ${ }^{4}$.

Considera-se a adolescência a fase que vai dos 10 aos 19 anos, período de maior vulnerabilidade para o uso de produtos derivados do tabaco, pois o indivíduo se encontra em processo de desenvolvimento físico, cognitivo e mental. Essa é uma fase de adaptação a mudanças orgânicas e da sexualidade, e o adolescente transita entre os limites da dependência infantil e a autonomia da fase adulta. Esse momento caracteriza-se pela construção da identidade e pela formulação do caráter social, ideológico e vocacional 5 .

Diante desse contexto, a atenção integral à saúde dos adolescentes requer um olhar diferente, com abordagem interdisciplinar, focada no sujeito e voltada às suas necessidades biopsicossociais e culturais, de maneira a facilitar a compreensão da complexidade do fenômeno humano e suas transformações, com o reconhecimento da família como base para a construção, socialização, humanização e integração com a comunidade 5

Quando se procura entender os motivos que levam um adolescente a se tornar fumante, é importante que sejam considerados tanto os fatores biológicos, como os sociais e psicológicos. O Ministério da Saúde brasileiro aponta que o tabagismo entre os jovens decorre da facilidade do acesso ao tabaco e das influências sociais, ambientais e genéticas 1. Além disso, o fato de as consequências negativas do consumo do tabaco só aparecerem tardiamente contribui para que os jovens fumem 6 .

Estudo de revisão sistemática aponta que fatores como curiosidade, tédio, estresse, influência de amigos e sensação de prazer e relaxamento também interferem na decisão de fumar nessa faixa etária. Pais fumantes também são conside- rados entre os principais fatores de influência para o hábito precoce - além do tabagismo passivo ao qual ficam expostos 6 .

Conhecer os fatores que influenciam o uso do tabaco é imprescindível para a elaboração de estratégias de prevenção, tratamento e redução dos índices de morbimortalidade relacionados ao uso desse produto. Estudos sobre a prevalência do tabagismo permitem que se avalie a dimensão desse problema na população e constituem uma importante ferramenta de diagnóstico da realidade para a adoção de medidas de controle 7 .

Assim, este estudo teve por objetivo avaliar o uso regular de cigarros industrializados por adolescentes e analisar sua relação com variáveis sociodemográficas, relações sociais e comportamentos de risco à saúde, procurando identificar os fatores associados ao início do uso do tabaco.

\section{Materiais e métodos}

Trata-se de estudo com delineamento epidemiológico transversal, realizado em escolas públicas da região urbana no Município de Londrina, Paraná, Brasil, no ano de 2011. A população foi composta por alunos matriculados no período diurno do 9o ano do Ensino Fundamental, série escolar que concentra a faixa etária entre 13 e 19 anos, faixa etária mais exposta ao início do tabagismo 8. Além disso, nessa idade, o aluno já apresenta melhor capacidade cognitiva, fator relevante para o preenchimento adequado do instrumento de coleta de dados. Desconsiderouse o período noturno, pois a maioria dos alunos desse turno pertence a uma faixa etária diferente e apresenta defasagem escolar, o que não caracterizaria o perfil proposto por esta pesquisa.

Para esse estudo, considerou-se um nível de 95\% de confiança. Como a precisão relativa em torno da estimativa é de $5 \%$, sendo a prevalência de $50 \%$, o que totalizou um tamanho amostral mínimo de 384 elementos, optou-se por utilizar uma amostra de 600 elementos.

Para a seleção dos elementos da amostra, foi utilizada amostragem equiprobabilística por estratificação, considerando, como estratos, as cinco regiões geográficas do município: norte, sul, leste, oeste e centro, o que resultou em uma amostra final de 517 participantes, pois 83 foram excluídos - 76 por não concordarem em participar da pesquisa, e sete por terem respondido incorretamente o instrumento de coleta de dados.

As informações foram coletadas por meio de um questionário baseado no estudo VIGESCOLA 9, que foi adaptado aos objetivos desta pesquisa, o que possibilitou uma comparação com a bibliografia disponível sobre o tema. 
O instrumento, composto por questões fechadas e de autopreenchimento e caracterizado como confidencial e não identificado, foi aplicado em sala de aula.

A variável dependente do estudo foi "uso regular de cigarros". A definição para "uso regular de cigarros" utilizada foi a mesma descrita nas principais pesquisas nacionais 8,9 : considera-se uso regular quando o indivíduo afirma ter fumado cigarros, pelo menos, um dia nos últimos trinta dias.

Para avaliar os fatores de risco associados ao uso regular de cigarros, as variáveis independentes foram selecionadas com base na literatura consultada e agrupadas, por afinidade, em dois blocos de covariáveis, sendo o primeiro constituído pelas variáveis sociodemográficas, e o segundo, pelas variáveis caracterizadas como relações sociais e de comportamentos de risco. Considerou-se, como estrutura básica familiar, a composta pelo pai e mãe, por ser essa a mais tradicional, em que cada membro possui papel definido 10 .

Os dados foram compilados no programa Microsoft Office Excel 2007 (Microsoft Corp., Estados Unidos) e, posteriormente, transportados para o programa SPSS, versão 15 (SPSS Inc., Chicago, Estados Unidos), no qual foram, inicialmente, analisados, de maneira descritiva ou por meio de cálculos de medidas estatísticas, com construção de tabelas e gráficos para a apresentação das informações mais relevantes.

Para avaliar a associação entre as variáveis independentes e a dependente, procedeu-se à análise univariada para se obter a razão de odds ratio (OR) com intervalo de 95\% de confiança (IC95\%). Foram selecionadas as variáveis independentes: sexo, idade, raça, classe social, escolaridade materna, religião, morar com o pai e a mãe, pais e/ ou irmãos fumantes, amigos fumantes, experimentação de álcool, atividade física e atividade remunerada. Posteriormente, realizou-se a análise multivariada, com o modelo de regressão logística e por meio do método stepwise forward, com as variáveis que apresentaram associação na análise univariada. Foi aceito o modelo que melhor explicou o uso regular de cigarros, com nível de significância de 0,05.

Este estudo foi aprovado pelo Comitê de Ética em Pesquisa Envolvendo Seres Humanos da Universidade Estadual de Londrina (CEP/UEL), sob CAAE no 0259.0.268.000-10. Participaram todos os alunos que, após convite, apresentaram o termo de consentimento livre e esclarecido (TCLE) devidamente assinado por eles e/ou seus responsáveis.

\section{Resultados}

Dos 517 estudantes participantes desta pesquisa, $69,8 \%(n=361)$ tinham idade igual ou inferior a 14 anos, sendo $48,7 \%(n=252)$ do sexo masculino e $51,3 \%(n=265)$ do feminino. Houve predominância das raças/cores branca $(51,3 \%, \mathrm{n}=265) \mathrm{e}$ parda $(37,9 \%, n=196)$. Entre os alunos, todas as classes sociais (Associação Brasileira de Empresas de Pesquisa. Critério de Classificação Econômica Brasil. http://www.abep.org/novo/Content. aspx?ContentID=302, acessado em 23/Mar/2012) estavam representadas, sendo a maior proporção pertencente às classes B1 e B2 $(63 \%, \mathrm{n}=324)$. Do total da população do estudo, 8,9\% $(\mathrm{n}=46)$ dos alunos informaram ter consumido cigarros nos últimos trinta dias, dos quais, $52,2 \%(n=24)$ eram meninos, e $47,8 \%(\mathrm{n}=22)$, meninas. Percentuais semelhantes de adolescentes fumantes (63\%; $n=29)$ e não fumantes $(62,2 \% ; n=293)$ apresentaram estrutura básica da composição familiar (Tabela 1).

A baixa escolaridade materna $(32,4 \% ; n=167)$ esteve presente entre todos os alunos pesquisados, e o percentual foi maior entre os usuários regulares de cigarros ( $37 \% ; \mathrm{n}=17$ ) (Figura 1). Vale destacar que, entre os usuários regulares de cigarros, identificou-se que $21,8 \%(n=10)$ das mães não concluíram o Ensino Fundamental; $15,2 \%(\mathrm{n}=7)$ apresentavam o Ensino Fundamental completo; 8,7\% ( $\mathrm{n}=4)$ não concluíram o Ensino Médio; $13 \%(n=6)$ terminaram o Ensino Médio; $10,9 \%(n=5)$ possuíam o Ensino Superior completo; e 30,4\% ( $n=14)$ dos adolescentes não souberam informar a escolaridade materna. Ressalta-se que, entre os 395 (76\%) adolescentes que informaram esses dados, $33 \%(n=131)$ eram filhos de analfabetas funcionais (escolaridade de até três anos de estudos completos) (Instituto Brasileiro de Geografia e Estatística. Taxa de Analfabetismo Funcional. http://seriesestatisticas.ibge. gov.br/series.aspx?vcodigo=PD384\&sv=8\&t=taxa-de-analfabetismo-funcional, acessado em 03/ Abr/2012).

O estudo também revelou um grande percentual de adolescentes expostos à fumaça tabágica, pois $35,6 \%(n=179)$ relataram ter pai, mãe e/ ou irmãos fumantes, e 47,6\% ( $n=246)$, amigos fumantes. A convivência com familiares tabagistas foi, percentualmente, igual entre os adolescentes fumantes e não fumantes. Por outro lado, entre os adolescentes que declararam ter amigos fumantes, a maioria fazia uso regular de cigarro (Tabela 2).

Em 82\% ( $n=429)$ das residências dos adolescentes entrevistados, não se permite o consumo de cigarros. Entretanto, 37\% ( $\mathrm{n}=192)$ informaram ser expostos à fumaça do cigarro dentro 
Perfil sociodemográfico dos estudantes do 9o ano do Ensino Fundamental de escolas públicas do Município de Londrina, Paraná, Brasil, 2011.

\begin{tabular}{|c|c|c|c|c|c|c|}
\hline \multirow[t]{3}{*}{ Características sociodemográficas } & \multicolumn{4}{|c|}{ Uso regular de cigarros * } & \multicolumn{2}{|c|}{ Total } \\
\hline & \multicolumn{2}{|c|}{ Sim } & \multicolumn{2}{|c|}{ Não } & \multirow[b]{2}{*}{$\mathrm{n}$} & \multirow[b]{2}{*}{$\%$} \\
\hline & $\mathrm{n}$ & $\%$ & $\mathrm{n}$ & $\%$ & & \\
\hline \multicolumn{7}{|l|}{ Sexo } \\
\hline Masculino & 24 & 52,2 & 228 & 48,5 & 252 & 48,7 \\
\hline Feminino & 22 & 47,8 & 243 & 51,5 & 265 & 51,3 \\
\hline \multicolumn{7}{|l|}{ Idade (anos) } \\
\hline 13 & 5 & 10,9 & 91 & 19,3 & 96 & 18,6 \\
\hline 14 & 17 & 37,0 & 248 & 52,7 & 265 & 51,2 \\
\hline 15 & 10 & 21,7 & 81 & 17,2 & 91 & 17,6 \\
\hline 16 ou mais & 14 & 30,4 & 51 & 10,8 & 65 & 12,6 \\
\hline \multicolumn{7}{|l|}{ Raça/Cor } \\
\hline Branca & 26 & 56,4 & 239 & 50,7 & 265 & 51,3 \\
\hline Preta & 1 & 2,2 & 39 & 8,3 & 40 & 7,7 \\
\hline Parda & 17 & 37,0 & 179 & 38,0 & 196 & 37,9 \\
\hline Amarela & 1 & 2,2 & 9 & 1,9 & 10 & 1.9 \\
\hline Indígena & 1 & 2,2 & 5 & 1,1 & 6 & 1,2 \\
\hline \multicolumn{7}{|l|}{ Classe social } \\
\hline$A(A 1, A 2)$ & 2 & 4,3 & 22 & 4,2 & 24 & 4,3 \\
\hline $\mathrm{B}(\mathrm{B} 1, \mathrm{~B} 2)$ & 25 & 54,4 & 299 & 63,9 & 324 & 63,0 \\
\hline$C(C 1, C 2)$ & 19 & 41,3 & 145 & 30,8 & 164 & 31,7 \\
\hline D & 0 & 0,0 & 4 & 0,9 & 4 & 0,8 \\
\hline$E$ & 0 & 0,0 & 1 & 0,2 & 1 & 0,2 \\
\hline \multicolumn{7}{|l|}{ Mora com o pai e a mãe } \\
\hline Sim & 29 & 63,0 & 293 & 62,2 & 322 & 62,3 \\
\hline Não & 17 & 37,0 & 178 & 37,8 & 125 & 37,7 \\
\hline \multicolumn{7}{|l|}{ Escolaridade materna } \\
\hline Baixa (menos de 9 anos) & 17 & 37,0 & 150 & 31,9 & 167 & 32,4 \\
\hline Alta (9 anos ou mais) & 15 & 32,6 & 213 & 45,2 & 228 & 44,1 \\
\hline Não souberam informar a escolaridade da mãe & 14 & 30,4 & 108 & 22,9 & 122 & 23,5 \\
\hline
\end{tabular}

* Relato de ter fumado cigarros, pelo menos, um dia nos últimos trinta dias anteriores à realização da pesquisa.

do domicílio, pelo menos, um dia da semana; a exposição diária ocorreu para $24 \%(n=122)$ dos adolescentes. Fora de casa, essa exposição também é diária para $22 \%(\mathrm{n}=115)$. A proibição ao fumo em locais públicos é apoiada por $90 \%$ ( $\mathrm{n}=467)$ dos adolescentes, e 93\% $(\mathrm{n}=479)$ concorda com a necessidade de solicitar permissão para fumar próximo a terceiros; apenas $33 \%$ (n = 169) permitiriam que alguém acendesse um cigarro ao seu lado.

Quanto à religião, apenas 7\% $(\mathrm{n}=36)$ são ateus. A maioria dos estudantes (93\%; $\mathrm{n}=481)$, portanto, segue alguma religião: 52\% ( $\mathrm{n}=269)$ católicos; 37,5\% ( $\mathrm{n}=194)$ evangélicos; e 3,5\% $(\mathrm{n}=18)$ divididos entre espíritas, adventistas, budistas, místicos, mórmons, testemunhas de Jeová e seguidores da Igreja de Cristo. Entre os fumantes, as porcentagens de adolescentes que referiram seguir alguma religião foram semelhantes às dos não fumantes (Tabela 2).

A maioria $(71 \% ; \mathrm{n}=367)$ dos entrevistados referiu não realizar atividades remuneradas; $12,4 \%$ ( $\mathrm{n}=64$ ) realizavam eventualmente; e apenas $16,6 \%(\mathrm{n}=86)$, habitualmente; esses percentuais não apresentaram diferenças entre os fumantes e os não fumantes. No entanto, a pesquisa apontou que todos os adolescentes dispunham de algum outro tipo de recurso financeiro, pois, entre os $84,5 \%(n=437)$ que responderam a questão: "Quanto tem em média para gastar no mês consigo mesmo?”, a maioria (66\%; $\mathrm{n}=289$ ) respondeu ter mais de $\mathrm{R} \$ 50,00 ; 23,8 \%(\mathrm{n}=104)$, entre 
Figura 1

Distribuição da escolaridade materna referida por escolares do 9o ano do Ensino Fundamental de escolas públicas do Município de Londrina, Paraná, Brasil, 2011.

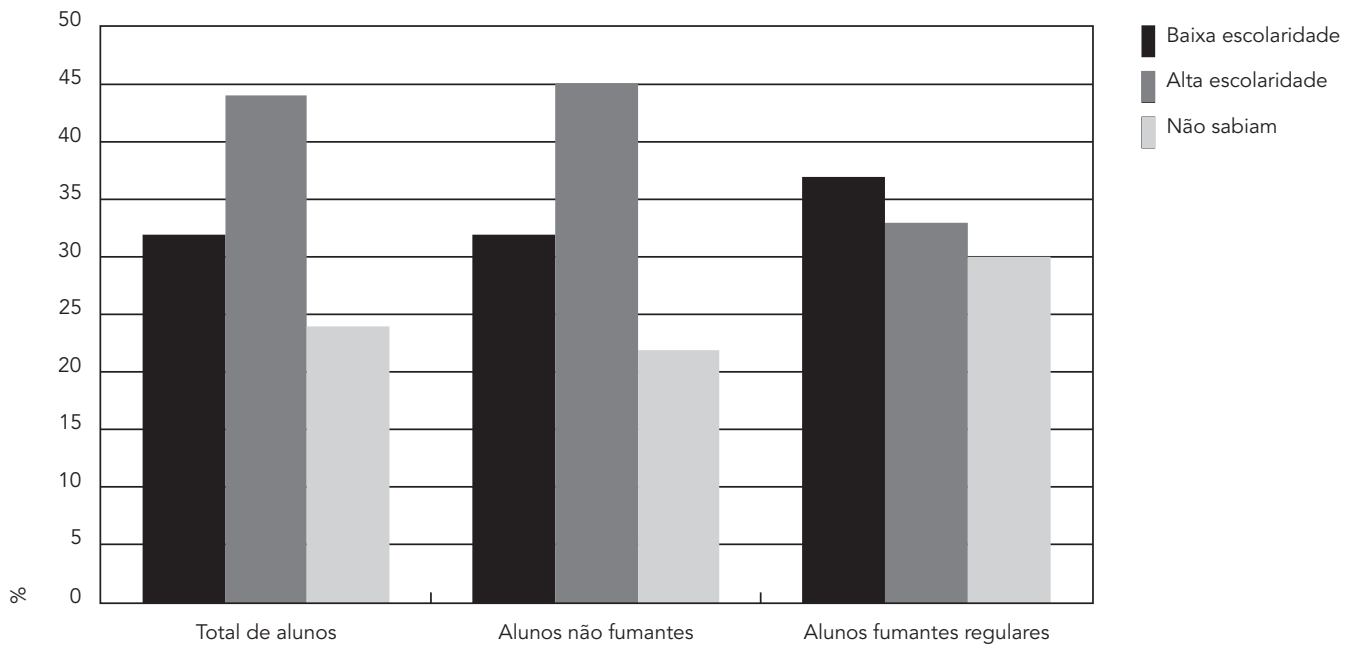

Tabela 2

Relações sociais e comportamentos de risco à saúde de estudantes do 9o ano do Ensino Fundamental de escolas públicas do Município de Londrina, Paraná, Brasil, 2011.

\begin{tabular}{|c|c|c|c|c|c|c|}
\hline \multirow[t]{3}{*}{ Relações sociais e comportamentos de risco à saúde } & \multicolumn{4}{|c|}{ Uso regular de cigarros * } & \multicolumn{2}{|c|}{ Total } \\
\hline & \multicolumn{2}{|c|}{ Sim } & \multicolumn{2}{|c|}{ Não } & \multirow[b]{2}{*}{$\mathbf{n}$} & \multirow[b]{2}{*}{$\%$} \\
\hline & $\mathrm{n}$ & $\%$ & $\mathrm{n}$ & $\%$ & & \\
\hline \multicolumn{7}{|l|}{ Pais ou irmãos fumantes } \\
\hline Sim & 16 & 34,8 & 163 & 34,7 & 179 & 35,6 \\
\hline Não & 30 & 65,2 & 308 & 65,3 & 338 & 65,4 \\
\hline \multicolumn{7}{|l|}{ Amigos fumantes } \\
\hline Sim & 42 & 91,1 & 204 & 43,3 & 246 & 47,6 \\
\hline Não & 4 & 8,9 & 267 & 56,7 & 271 & 52,4 \\
\hline \multicolumn{7}{|l|}{ Religião } \\
\hline $\operatorname{Sim}$ & 44 & 95,6 & 437 & 92,8 & 481 & 93,0 \\
\hline Não & 2 & 4,4 & 34 & 7,2 & 36 & 7,0 \\
\hline \multicolumn{7}{|l|}{ Atividade remunerada } \\
\hline Sim & 15 & 32,6 & 135 & 28,7 & 150 & 29,0 \\
\hline Não & 31 & 67,4 & 336 & 71,3 & 367 & 71,0 \\
\hline \multicolumn{7}{|l|}{ Atividade física } \\
\hline Sim & 24 & 52,2 & 276 & 58,6 & 300 & 58,0 \\
\hline Não & 22 & 47,8 & 195 & 41,4 & 217 & 42,0 \\
\hline \multicolumn{7}{|l|}{ Experimentação de álcool } \\
\hline Sim & 44 & 95,6 & 418 & 88,7 & 462 & 89,4 \\
\hline Não & 2 & 4,4 & 53 & 11,3 & 55 & 10,6 \\
\hline
\end{tabular}

* Relato de ter fumado cigarros, pelo menos, um dia nos últimos trinta dias anteriores à realização da pesquisa. 
$\mathrm{R} \$ 10,00$ e $\mathrm{R} \$ 50,00 ; 5,2 \%(\mathrm{n}=23)$ menos que $\mathrm{R} \$$ 10,00 ; e somente $5 \%(n=21)$ afirmaram não ter nenhum recurso. Como um maço de cigarros pode ser comprado por um valor médio de $\mathrm{R} \$ 4,00$, pode-se considerar que grande parte dos adolescentes pesquisados poderia utilizar seu dinheiro para ter acesso ao cigarro, se assim o desejasse.

Esta pesquisa mostrou que a prática de atividade física ainda não estava inserida no cotidiano dos adolescentes pesquisados, pois $42 \%$ ( $\mathrm{n}=$ 217) referiram não realizar nenhuma atividade física fora do horário de aula; 29,6\% ( $\mathrm{n}=153)$ realizavam até 2 vezes por semana; 14,9\% ( $\mathrm{n}=77)$, de 3 a 4 vezes; e somente $13,5 \%(n=70)$, mais de 5 vezes por semana.

A experimentação de álcool pelos adolescentes participantes deste estudo foi muito elevada, pois a maioria $(89,4 \% ; n=462)$ informou ter experimentado algum tipo de bebida alcoólica, e $47 \%(n=241)$ referiram ter consumido bebida alcoólica nos últimos trinta dias. Entre os adolescentes usuários regulares de cigarro, o percentual de experimentação foi ainda maior (95,6\%; $\mathrm{n}=44)$, sendo que $72 \%(\mathrm{n}=33)$ deles haviam consumido bebida alcoólica nos últimos trinta dias.
Na Tabela 3, são apresentados os resultados da análise univariada entre o uso regular de cigarros e as características sociodemográficas. Nessa análise, apenas a idade apresentou associação estatística $(\mathrm{p}=0,001)$. Adolescentes com 15 anos ou mais apresentaram 2,8 vezes mais chances de serem usuários regulares de cigarros que aqueles com até 14 anos (OR = 2,80; IC95\%: 1,52-5,17).

A Tabela 4 apresenta os resultados da análise univariada entre o uso regular de cigarros e as relações sociais e os comportamentos de risco. Observou-se que ter pais ou irmãos fumantes, seguir alguma religião, praticar atividade física, ter experimentado álcool e realizar atividade remunerada não apresentaram associação. No entanto, ter amigos fumantes (OR=13,74; IC95\%: 4,85-38,94) constituiu-se em fator de risco.

$\mathrm{Na}$ análise multivariada, a idade $\geq 15$ anos ( $\mathrm{OR}=2$,28; IC95\%: 1,21-4,32; $\mathrm{p}=0,011$ ) e possuir amigos fumantes (OR = 12,62; IC95\%: 4,44-35,89; $\mathrm{p}<0,0001$ ) mantiveram-se como fatores de risco para o uso regular de cigarros dos adolescentes.

\begin{tabular}{|c|c|c|c|}
\hline Fatores de risco & OR (bruta) & IC95\% & Valor de $p$ \\
\hline \multicolumn{4}{|l|}{ Sexo } \\
\hline Masculino & 1,00 & & 0,62 \\
\hline Feminino & 0,86 & $0,47-1,58$ & \\
\hline \multicolumn{4}{|l|}{ Idade (anos) } \\
\hline$\leq 14$ & 1,00 & & 0,001 \\
\hline$\geq 15$ & 2,80 & $1,52-5,17$ & \\
\hline \multicolumn{4}{|l|}{ Raça/Cor } \\
\hline Branca & 1,00 & & 0,45 \\
\hline Outras & 0,79 & $0,43-1,46$ & \\
\hline \multicolumn{4}{|l|}{ Classe social } \\
\hline Alta (A e B) & 1,00 & & 0,19 \\
\hline Baixa (C, D e E) & 1,51 & $0,81-2,79$ & \\
\hline \multicolumn{4}{|c|}{ Morar com os pais (pai e a mãe) } \\
\hline Sim & 1,00 & & 0,86 \\
\hline Não & 1,06 & $0,56-1,98$ & \\
\hline \multicolumn{4}{|c|}{ Escolaridade materna * } \\
\hline Alta & 1,00 & & 0,19 \\
\hline Baixa & 1,61 & $0,78-3,32$ & \\
\hline
\end{tabular}

IC95\%: intervalo de $95 \%$ de confiança; OR: odds ratio.

${ }^{*} \mathrm{n}=395$ 
Associação entre o uso regular de cigarros e relações sociais e comportamentos de risco à saúde em estudantes do 9o ano do Ensino Fundamental de escolas públicas do Município de Londrina, Paraná, Brasil, 2011.

\begin{tabular}{|c|c|c|c|}
\hline Relações sociais e comportamentos de risco à saúde & OR (bruta) & (IC95\%) & Valor de $\mathrm{p}$ \\
\hline \multicolumn{4}{|l|}{ Pais ou irmãos fumantes } \\
\hline Não & 1,00 & & 0,98 \\
\hline Sim & 1,01 & $0,53-1,90$ & \\
\hline \multicolumn{4}{|l|}{ Amigos fumantes } \\
\hline Não & 1,00 & & $<0,0001$ \\
\hline Sim & 13,74 & $4,85-38,94$ & \\
\hline \multicolumn{4}{|l|}{ Experimentação de álcool } \\
\hline Não & 1,00 & & 0,16 \\
\hline $\operatorname{Sim}$ & 2,79 & $0,66-11,84$ & \\
\hline \multicolumn{4}{|l|}{ Religião } \\
\hline Sim & 1,00 & & 0,46 \\
\hline Não & 1,72 & $0,40-7,40$ & \\
\hline \multicolumn{4}{|l|}{ Atividade física } \\
\hline Sim & 1,00 & & 0,39 \\
\hline Não & 1,30 & $0,71-2,39$ & \\
\hline \multicolumn{4}{|l|}{ Atividade remunerada } \\
\hline Não & 1,00 & & 0,58 \\
\hline Sim & 1,19 & $0,63-2,29$ & \\
\hline
\end{tabular}

IC95\%: intervalo de 95\% de confiança; OR: odds ratio.

\section{Discussão}

Este estudo identificou duas variáveis importantes associadas ao uso regular de cigarros entre adolescentes: idade e ter amigos fumantes. A idade de 15 anos ou mais se constituiu fator de risco. Tais resultados são compatíveis com outras pesquisas longitudinais que revelaram que, quanto mais velho o adolescente for, maior será o risco para o uso de produtos derivados do tabaco $8,11,12,13,14$.

Um dos principais resultados deste estudo aponta para a relação entre possuir amigos fumantes e o uso regular de cigarros entre os adolescentes. Esses dados concordam com os de outros estudos realizados no Brasil 12,15,16,17 e nos Estados Unidos 14,18,19,20, entre outros países $21,22,23,24$. Pesquisa que avaliou, qualitativamente, os fatores socioculturais associados à iniciação $\mathrm{e}$ cessação do uso do tabaco mostrou que a pressão dos amigos foi um dos mais importantes fatores de influência para a iniciação e uso regular do cigarro 25 . Em um estudo longitudinal utilizando uma amostra representativa da população de adolescentes nos Estados Unidos, ter amigos fumantes foi o fator de risco mais importante para a iniciação do uso do tabaco comparado às outras variáveis estudadas 14 .
O resultado deste estudo, ao demonstrar que o uso regular de cigarros entre os adolescentes não apresentou associação com a variável "morar com o pai e a mãe", diverge de outros estudos que avaliaram a estrutura familiar como variável importante para o tabagismo adolescente e que apresentaram resultados que evidenciam a associação entre o uso do tabaco e a instabilidade do relacionamento dos pais, observando maior risco para o uso do tabaco entre os adolescentes com pais separados 11,26.

Os resultados divergentes quanto à variável estrutura familiar não significam que se negue, aqui, o papel da família na construção da personalidade do adolescente, pois é no contexto familiar que são transmitidos os valores, as crenças e os aspectos culturais e cognitivos, por meio da relação entre pais e filhos. Esses fatores, que podem sofrer influências sociais, contribuem para o desenvolvimento da personalidade do indivíduo 27 . Ao longo do tempo, entretanto, as famílias sofreram alterações em sua composição, e, atualmente, as configurações são variadas, tais como: só mãe ou pai; pais adotivos; famílias combinadas, entre outras, cujos membros, muitas vezes, não têm papéis bem definidos - o que pode tornar o ambiente familiar disfuncional e, nesse caso, impactar negativamente a formação 
do indivíduo 10,27. Por outro lado, já não se pode afirmar que uma composição familiar diferente da tradicional necessariamente desencadeie efeitos nocivos sobre seus componentes. Nessa perspectiva, modelos positivos também ocorrem, pois indivíduos que vivem em situações adversas podem criar mecanismos de superação e desenvolver uma vida saudável, numa atitude de resiliência 28 .

Estudo prévio identificou a influência do grau de escolaridade materna no início da utilização do tabaco por parte dos filhos adolescentes, isto é, quanto menor o nível de escolaridade da mãe, maior a incidência de experimentação de cigarros 11. Na população deste estudo, a baixa escolaridade materna não apresentou associação com o uso regular do cigarro. Esse resultado corrobora outros estudos, que também não encontraram essa associação 8,26,29.

A escolaridade materna pode ser considerada como fator positivo no desenvolvimento saudável do indivíduo. A mãe com maior nível de escolaridade pode assimilar, com mais facilidade, as orientações recebidas e, consequentemente, as transmitirá com mais clareza aos filhos. Um estudo que avaliou a qualidade dos estímulos e o desempenho cognitivo de 350 crianças de até cinco anos comprovou a relação entre um maior grau na escolaridade materna e a melhor qualidade dos estímulos dispensados à criança, impactando positivamente no desempenho cognitivo 30 .

$\mathrm{O}$ fato de ter pais ou irmãos fumantes não apresentou associação ao uso regular de cigarros pelos adolescentes pesquisados, resultado que corroborou dados levantados por um estudo realizado por Zanini et al. 12. Trata-se de resultado muito díspar de inúmeras outras pesquisas, que identificaram a influência dos pais e irmãos fumantes sobre o hábito de fumar do adolescente $15,16,17,19$. Cordeiro et al. 31, por exemplo, apontaram que ter ambos os pais fumantes dobra o risco de experimentação de cigarro.

Esta pesquisa mostrou que a prática de atividade física ainda não está inserida no cotidiano dos adolescentes pesquisados, pois muitos referiram não realizar nenhuma atividade física fora do horário de aula. A atividade física pode ser considerada como um fator de proteção à saúde e contra comportamentos de risco, dentre eles, o uso do tabaco em idade precoce 8 .

A religião, a atividade física e a atividade remunerada não foram estatisticamente associadas ao uso regular de cigarros. Esses dados diferem dos levantados por outros estudos. Barreto et al. ${ }^{8}$ verificaram associação protetora entre o uso do tabaco e a prática de atividade física. Silva et al. 11 identificaram que o risco de experimentar o tabaco é maior entre os que não realizam atividade física, os que não têm religião e os que possuem alguma forma de remuneração. Em um estudo entre 4.746 alunos em escolas de Ensino Médio no Estado de Minnesota, nos Estados Unidos, observou-se uma relação inversa entre o uso do tabaco e atividade física 32 .

O percentual de adolescentes que havia experimentado álcool foi maior do que o apresentado pelo Centro Brasileiro de Informações sobre Drogas Psicotrópicas (CEBRID), em levantamento realizado em 27 capitais brasileiras no ano de $2004(65,2 \%) 33$. Entre os adolescentes usuários regulares de cigarros, o percentual de experimentação foi relativamente ainda maior. No entanto, no presente estudo, não foi encontrada associação estatística da experimentação do álcool com o uso regular de cigarros. Outras pesquisas identificaram associação entre os comportamentos de fumar e o uso de álcool 8,16,33,34,35.

Entre as limitações deste estudo, ressalta-se o fato de a pesquisa ter sido realizada somente em escolas públicas e com alunos do $9 \underline{\text { a }}$ ano do período diurno, portanto, outros dados poderiam ser encontrados caso fossem incluídos escolas privadas, alunos de outras faixas etárias e do período noturno, o que aponta para a necessidade de desenvolvimento de outras pesquisas que analisem outras populações.

\section{Conclusão}

As razões que levam o adolescente a fumar são específicas da idade e diferentes das apresentadas pelos adultos, e o abandono do tratamento é uma das principais dificuldades para a cessação do tabagismo na adolescência. Portanto, é fundamental o acompanhamento de grupos exclusivos que permitam a percepção e a compreensão dos fatores associados ao uso do tabaco na adolescência, com o objetivo de alcançar maior adesão às ações programáticas 36 .

O uso do tabaco na adolescência, assim como de outras drogas, afeta as condições de saúde e a qualidade de vida dos jovens. Assim, é necessário o desenvolvimento de mecanismos de proteção, prevenção e acolhimento que deem suporte à curiosidade inerente a essa fase da vida, por meio da implementação de políticas públicas que estimulem escolhas de vida saudáveis e de qualidade, desmistificando a falsa ideia de autonomia propiciada por substâncias tóxicas 5 .

Nesta pesquisa, constituíram-se em fatores de risco para o uso regular de cigarros entre os adolescentes investigados: possuir a idade igual ou superior a 15 anos e ter amigos fumantes. O segundo fator, ao lado de resultados de estudos realizados em diferentes culturas, alertam para 
a importância de educadores e pais estarem vigilantes, procurando conhecer os amigos dos filhos, para apoiá-los na manutenção das influências positivas e na superação das negativas 14,33.

Os resultados obtidos apontam para a necessidade do planejamento e implementação de programas de promoção à saúde escolar, com o incremento de ações antitabágicas direcionadas a adolescentes, que possibilitem a obtenção de indicadores positivos, por meio de parcerias intersetoriais que envolvam as entidades educacionais, a família e a sociedade como um todo.

\section{Resumen}

Estudio epidemiológico con el objetivo de identificar los factores asociados al consumo de cigarrillos por parte de adolescentes. La muestra estuvo formada por 517 estudiantes de 9o curso de primaria en Londrina, Paraná, Brasil. En el análisis estadístico se utilizó el modelo de regresión logística, estableciendo un nivel de significación del 5\%. Se informó del consumo de cigarrillos por lo menos un día, durante los últimos treinta días, en un 8,9\% de los adolescentes. Los factores de edad $\geq 15$ años $(O R=2,28 ;$ IC95\%: 1,21-4,32; $p=0,011)$ y tener amigos fumadores $(O R=12,62$; IC95\%: 4,44-35,89; $p<0,0001)$ se asociaron con el consumo regular de cigarrillos, tanto en el uni-, como en el análisis multivariante. Por otro lado, el sexo, la raza, la clase social, la convivencia con el padre y la madre, la religión, la educación materna, la experimentación con el alcohol, la actividad física y la actividad remunerada no están asociados con esta práctica. Estos hallazgos pueden apoyar en el desarrollo de estrategias de control del consumo del tabaco dirigidas a la comunidad.

Hábito de Fumar; Tabaco; Estudiantes; Salud del Adolescente

\section{Colaboradores}

A. H. R. Menezes, A. A. M. Cardelli e J. C. Dalmas participaram da concepção do projeto, da análise dos dados e da revisão crítica relevante do conteúdo intelectual. I. C. Scarinci colaborou na análise e interpretação dos dados e revisão crítica do conteúdo intelectual. S. M. Maciel contribuiu em todo processo de revisão do artigo, particularmente, na correção dos procedimentos estatísticos.

\section{Agradecimentos}

Aos professores do Curso de Mestrado de Enfermagem da Universidade Estadual de Londrina. 


\section{Referências}

1. Instituto Nacional de Câncer. Jovens e mulheres na mira da indústria do tabaco. http://www. inca.gov.br/tabagismo/frameset.asp?item=jovem \&link=namira.htm (acessado em 27/Mar/2012).

2. Cinciripini PM, Hecht SS, Henningfield JE, Manley MW, Kramer BS. Tobacco addiction: implications for treatment and cancer prevention. J Natl Cancer Inst 1997; 89:1852-67.

3. Scivoletto S. Abordagem de populações especiais: adolescentes. In: Gigliotti AP, Presman S, organizadores. Atualização no tratamento do tabagismo. Rio de Janeiro: ABP-Saúde; 2006. p. 135-45.

4. Instituto Brasileiro de Geografia e Estatística. Pesquisa Nacional por Amostra de Domicílios (PNAD): tabagismo, 2008. Rio de Janeiro: Instituto Brasileiro de Geografia e Estatística; 2009.

5. Maakaroum MF, Souza RCP. A consulta do adolescente. In: Lopez FA, Campos-Júnior D, organizadores. Tratado de pediatria: Sociedade Brasileira de Pediatria. Barueri: Edições Manole; 2007. p. 341-7.

6. Moolchan E, Ernst M, Henningfield JE. A review of tobacco smoking in adolescents: treatment implications. J Am Acad Child Adolesc Psychiatry 2000; 39:682-93.

7. Hallal ALL. Fatores associados ao tabagismo em escolares da Região Sul do Brasil [Tese de Doutorado]. São Paulo: Faculdade de Saúde Pública, Universidade de São Paulo; 2008.

8. Barreto SM, Giatti L, Casado L, Moura L, Crespo C, Malta DC. Exposição ao tabagismo entre escolares no Brasil. Ciênc Saúde Coletiva 2010; 15 Suppl 2:3027-34

9. Instituto Nacional de Câncer. VIGESCOLA: Vigilância de Tabagismo em Escolares. Dados e fatos em 12 capitais brasileiras. Rio de Janeiro: Instituto Nacional de Câncer; 2004.

10. Pratta EMM, Santos MA. Família e adolescência: a influência do contexto familiar no desenvolvimento psicológico de seus membros. Psicol Estud 2007; 12:247-56

11. Silva MP, Silva RMVG, Botelho C. Fatores associados à experimentação do cigarro em adolescentes. J Bras Pneumol 2008; 34:927-35.

12. Zanini RR, Moraes AB, Trindade ACA, Riboldi J, Medeiros LR. Prevalência e fatores associados ao consumo de cigarros entre estudantes de escolas estaduais do ensino médio de Santa Maria, Rio Grande do Sul, Brasil, 2002. Cad Saúde Pública 2006; 22:1619-27.

13. Malcon MC, Menezes AMB, Chatkin M. Prevalência e fatores de risco para tabagismo em adolescentes. Rev Saúde Pública 2003; 37:1-7.

14. Mahabee-Gittens EM, Xiao Y, Gordon JS, Khoury JC. The dynamic role of parental influences in preventing adolescent smoking initiation. Addict Behav 2013; 38:1905-11.

15. Pinto DS, Ribeiro AS. Variáveis relacionadas à iniciação do tabagismo entre estudantes do ensino médio de escola pública e particular na cidade de Belém - PA. J Bras Pneumol 2007; 33:558-64.
16. Vieira PC, Aerts DRGC, Freddo SL, Bittencourt A, Monteiro L. Uso de álcool, tabaco e outras drogas por adolescentes escolares em município do Sul do Brasil. Cad Saúde Pública 2008; 24:2487-98.

17. Oliveira HF, Martins LC, Reato LFN, Akerman M. Fatores de risco para uso do tabaco em adolescentes de duas escolas do Município de Santo André, São Paulo. Rev Paul Pediatr 2010; 28:200-7.

18. Bernat DH, Klein EG, Forster JL. Smoking initiation during young adulthood: a longitudinal study of a population-based cohort. J Adolesc Health 2012; 51:497-502.

19. Goldade K, Choi K, Bernat DH, Klein EG, Okuyemi KS, Forster J. Multilevel predictors of smoking initiation among adolescents: findings from the Minnesota Adolescent Community Cohort (MACC) study. Prev Med 2012; 54:242-6.

20. Stanton CA, Papandonatos G, Lloyd-Richardson EE, Kazura A, Shiu SY, Niaura R. How do mothers, fathers, and friends influence stages of adolescent smoking? Adolesc Fam Health 2009; 4:95-111.

21. Nwafor CC, Ibeh CC, Aguwa EN, Chukwu JN. Assessment of pattern of cigarette smoking and associated factors among male students in public secondary schools in Anambra State, Nigeria. Niger J Med 2012; 21:41-7.

22. Reda AA, Moges A, Yazew B, Biadgilign S. Determinants of cigarette smoking among school adolescents in eastern Ethiopia: a cross-sectional study. Harm Reduct J 2012; 9:39.

23. Morello P, Duggan A, Adger Jr. H, Anthony JC, Joffe A. Tobacco use among high school students in Buenos Aires, Argentina. Am J Public Health 2001; 91:219-24.

24. Tjora T, Hetland J, Aaro LE, Overland S. Distal and proximal family predictors of adolescents' smoking initiation and development: a longitudinal latent curve model analysis. BMC Public Health 2011; 11:911.

25. Scarinci IC, Silveira AF, Santos DF, Beech BM. Sociocultural factors associated with cigarette smoking among women in Brazilian worksites: a qualitative study. Health Promot Int 2007; 22:146-54.

26. Horta BL, Calheiros P, Pinheiro RT, Tomasi E, Amaral KC. Tabagismo em adolescentes de área urbana na região Sul do Brasil. Rev Saúde Pública 2001; 35:159-64.

27. Sauer MTN, Eisenstein E, Quadros JC. Sexualidade na adolescência. In: Lopez FA, Campos-Júnior D, organizadores. Tratado de pediatria: Sociedade Brasileira de Pediatria. Barueri: Edições Manole; 2007. p. 365-73.

28. Sapienza G, Pedromônico MRM. Risco, proteção e resiliência no desenvolvimento da criança e do adolescente. Psicol Estud 2005; 10:209-16.

29. Fraga S, Ramos E, Barros H. Uso de tabaco por estudantes adolescentes portugueses e fatores associados. Rev Saúde Pública 2006; 40:620-6. 
30. Andrade SA, Santos DN, Bastos AC, Pedromônico MRM, Almeida-Filho N, Barreto ML. Ambiente familiar e desenvolvimento cognitivo infantil: uma abordagem epidemiológica. Rev Saúde Pública 2005; 39:606-11.

31. Cordeiro EAK, Kupek E, Martini JG. Prevalência do tabagismo entre escolares de Florianópolis, SC, Brasil e as contribuições da enfermagem. Rev Bras Enferm 2010; 63:706-11.

32. Larson NI, Story M, Perry CL, Neumark-Sztainer D, Hannan PJ. Are diet and physical activity patterns related to cigarette smoking in adolescents? Findings from Project EAT. Prev Chronic Dis 2007; 4:A51.

33. Schenker M, Minayo MCS. Fatores de risco e de proteção para o uso de drogas na adolescência. Ciênc Saúde Coletiva 2005; 10:707-17.
34. Rodrigues MC, Viegas CAA, Gomes EL, Morais JPMG, Zakir JCO. Prevalência do tabagismo e associação com o uso de outras drogas entre escolares do Distrito Federal. J Bras Pneumol 2009; 35 986-91.

35. Leatherdale ST, Hammond D, Ahmed R. Alcohol, marijuana, and tobacco use patterns among youth in Canada. Cancer Causes Control 2008; 19:361-9.

36. Reichert J, Araújo AJ, Gonçalves CC, Godoy I, Chatkin JM, Sales MPU, et al. Diretrizes para cessação do tabagismo - 2008. J Bras Pneumol 2008; 34:845-80.

Recebido em 04/Dez/2012

Versão final reapresentada em 26/Jun/2013

Aprovado em 14/Out/2013 